\section{True Difference or Detection Bias: Racial Differences in Clinical Features and Comorbidities in Ankylosing Spondylitis in the United States}

\section{To the Editor:}

We read with interest about the study by Singh and Magrey ("Racial differences in clinical features and comorbidities in ankylosing spondylitis in the United States") ${ }^{1}$. The authors selected patients with ankylosing spondylitis (AS) from a large clinical informatics tool, the Explorys platform, with multiple participating healthcare organizations, which increases the generalizability of the findings. To increase the validity of the cases, Singh and Magrey included AS patients with at least 2 visits with a rheumatologist. While this approach may increase case validity, it might miss patients who have not seen a rheumatologist or were lost to followup after the initial visit. Only $8 \%$ of the patients included in the study were African American. It is unclear whether such a low prevalence is from low detection in these patients or due to a true racial advantage. Factors such as socioeconomic status, access to care, and misdiagnosis might have led to the low representation in this population. Further, it is possible that the cases diagnosed in earlier years (for example, 1999 to 2010) might have missed more ethnic populations, because traditionally this was thought to be a disease primarily of the white population.

Singh and Magrey performed a subgroup analysis at their institution that showed that only $42 \%$ of African Americans were HLA-B27-positive, compared to up to $90 \%$ of the whites ${ }^{2}$. This is slightly lower than the previously reported frequency of HLA-B27 in the African American population (between $50 \%$ and $60 \%)^{3,4,5}$. A higher proportion of extraarticular manifestations and typical presentations such as enthesopathy, dactylitis, or inflammatory bowel disease was seen in African Americans. This again points to the possibility of a detection bias because only those African American patients with these typical features and HLA-B27 positivity might have been diagnosed, and others with more subtle symptoms might have been missed. Similarly, differences in comorbidities between the groups might be secondary to a higher index of suspicion for AS in whites, leading to detection of milder cases in them. The unconscious bias of physicians that AS is specific to traditional HLA-B27-positive whites could play a role. Further, a higher percentage of African American patients had elevated inflammatory markers than did whites, which could again be a function of increased AS diagnosis in African American patients with higher inflammatory markers. It would not be surprising to see a higher prevalence of comorbidities such as hypertension, diabetes, depression, and heart disease in this subgroup with increased inflammatory burden.

The differences in this study between the African American and white populations might be related to various types of detection bias rather than a true difference in phenotype. It is possible that a higher proportion of African American patients with less severe disease failed to be diagnosed with AS compared to whites. Alternatively, delayed diagnosis might have led to a higher inflammatory burden. Higher disease activity was also seen in the African American patients in the Prospective Study of Outcomes in Ankylosing Spondylitis cohort, even after controlling for disease duration, sex, smoking status, education levels, and medications ${ }^{5}$. However, disease duration and symptom duration are often very different from each other, and it is usually not possible to find information regarding symptom duration in administrative databases. Similarly, the study does not include patients with nonradiographic axial spondyloarthritis (nr-axSpA). The prevalence of nraxSpA in the African American population is somewhat unclear, and it is possible that this population has a relatively high prevalence of nr-axSpA. Detection bias may play a bigger role in nr-axSpA because it is often diagnosed only with advanced techniques such as magnetic resonance imaging, access to which might be limited in this population. Further, a lower percentage of women with nr-axSpA was noted in Northwest Africa compared to Southern Africa ( $42 \%$ vs $57 \%$ ), which could relate to a lower likelihood of women seeking care in that region ${ }^{6}$. Therefore, a populationbased study with adequate representation of African American patients might provide better insight into the racial prevalence and clinical features of axSpA in this population.

PARAS KARMACHARYA @ , MBBS, Division of Rheumatology, Mayo Clinic; JOYCE E. BALLS-BERRY (D), PhD, Mayo Clinic College of Medicine and Science, Department of Health Sciences Research, Mayo Clinic; JOHN M. DAVIS III $『$, MD, MS, Division of Rheumatology, Mayo Clinic, Rochester, Minnesota, USA. Address correspondence to P. Karmacharya, Division of Rheumatology, Mayo Clinic College of Medicine, 200 First St. S.W., Rochester, Minnesota 55905, USA. E-mail: paraskarmacharya@gmail.com

\section{REFERENCES}

1. Singh DK, Magrey MN. Racial differences in clinical features and comorbidities in ankylosing spondylitis in the United States. J Rheumatol 2020;47:835-8.

2. Cortes A, Pulit SL, Leo PJ, Pointon JJ, Robinson PC, Weisman MH, et al. Major histocompatibility complex associations of ankylosing spondylitis are complex and involve further epistasis with ERAP1. Nat Commun 2015;6:7146.

3. Ouédraogo DD, Tiéno H, Kaboré H, Palazzo E, Meyer O, Drabo JY. Ankylosing spondylitis in rheumatology patients in Ouagadougou (Burkina Faso). Clin Rheumatol 2009;28:1375-7.

4. Khan MA, Kushner I, Braun WE. Low incidence of HLA-B27 in American Blacks with spondyloarthropathies. Lancet 1976;1:483.

5. Jamalyaria F, Ward MM, Assassi S, Learch TJ, Lee M, Gensler LS, et al. Ethnicity and disease severity in ankylosing spondylitis a cross-sectional analysis of three ethnic groups. Clin Rheumatol 2017;36:2359-64.

6. Shirazy K, Hajjaj-Hassouni N, Hammond C, Jones H, Ladjouze Rezig A, Pedersen R, et al. The prevalence of non-radiographic axial spondyloarthritis among patients with inflammatory back pain from Northwest and South Africa: data from a noninterventional, cross-sectional study. Rheumatol Ther 2018;5:437-45.

First Release February 15 2020; J Rheumatol 2020;47:7;

doi:10.3899/jrheum.191399 